\title{
Very high energy emission from the hard spectrum sources HESS J1641-463, HESS J1741-302 and HESS J1826-130
}

\section{E. O. Angüner ${ }^{* a}$, S. Casanova ${ }^{a, b}$, I. Oya ${ }^{c}$, F. Aharonian ${ }^{b, d}$, P. Bordas ${ }^{b}$ and A. Ziegler for the H.E.S.S. Collaboration.}

a Instytut Fizyki Jadrowej PAN, ul. Radzikowskiego 152, 31-342 Kraków, Poland

${ }^{b}$ Max-Planck-Institute fr Kernphysik, P.O. Box 103980, D 69029 Heidelberg, Germany

${ }^{c}$ DESY, Platanenallee 6, D-15738 Zeuthen, Germany

${ }^{d}$ Dublin Institute for Advanced Studies, 31 Fitzwilliam Place, Dublin 2, Ireland

${ }^{e}$ Friedrich-Alexander-Universität Erlangen-Nürnberg, Erlangen Centre for Astroparticle Physics, Erwin-Rommel-Str. 1, D 91058 Erlangen, Germany

E-mail: oguzhan.angunereifj.edu.pl,

sabrina.casanova@mpi-hd.mpg.de,

igor.oya.vallejoddesy.de,

felix.aharonian@mpi-hd.mpg.de,

pol.bordas@mpi-hd.mpg.de,

alexander.zieglerefau.de

A recent study of the diffuse $\gamma$-ray emission in the Central Molecular Zone using very high energy (VHE, E $>0.1 \mathrm{TeV}$ ) H.E.S.S. data suggests that the Galactic Center (GC) is the most plausible supplier of Galactic ultra-relativistic cosmic-rays (CRs) up to the knee at about $10^{15} \mathrm{eV}(\mathrm{PeV})$. However, the GC might not be the only source capable to accelerate CRs up to PeV energies in the Galaxy. Here we present H.E.S.S. data analysis results and interpretation of three H.E.S.S. sources, with spectra extending beyond $10 \mathrm{TeV}$ and relatively hard spectral indices compared with the average spectral index of H.E.S.S. sources, namely HESS J1641-463, HESS J1741-302 and HESS J1826-130. Although the nature of these VHE $\gamma$-ray sources is still open, their spectra suggest that the astrophysical objects producing such emission must be capable of accelerating the parental particle population up to energies of at least several hundreds of TeV. Assuming a hadronic scenario, dense gas regions can provide rich target material for accelerated particles to produce VHE $\gamma$-ray emission via proton-proton interactions followed by a subsequent $\pi^{0}$ decay. Thus, detailed investigations of the interstellar medium along the line of sight to all of these sources have been performed by using data from available atomic and molecular hydrogen surveys. The results point out the existence of dense interstellar gas structures coincident with the best fit positions of these sources. One can find possible hadronic models with CRs being accelerated close to the $\mathrm{PeV}$ energies to explain the $\gamma$-ray emission from all of these sources, which opens up the possibility that a population of $\mathrm{PeV} \mathrm{CR}$ accelerators might be active in the Galaxy.

35th International Cosmic Ray Conference - ICRC2017

10-20 July, 2017

Bexco, Busan, Korea

${ }^{*}$ Speaker. 


\section{INTRODUCTION}

Investigation of the diffuse very high energy (VHE, $\mathrm{E}>0.1 \mathrm{TeV}$ ) H.E.S.S. $\gamma$-ray data around the Galactic Centre (GC) region suggests the presence of $\mathrm{PeV}\left(10^{15} \mathrm{eV}\right)$ particles within the central 10 parsec of the Galaxy, making the GC the most plausible supplier of ultra-relativistic cosmic-rays (CRs), a "PeVatron" [1]. However, there may be other sources contributing to the Galactic CR flux around the knee, representing a new source population. One expects that such sources, capable of accelerating protons (hadrons) up PeV energies, should have relatively hard spectral indices without showing any cut-off in their VHE $\gamma$-ray spectra. The H.E.S.S. Galactic Plane Scan (HGPS) catalog [2] can be used to pin-point sources with spectra extending beyond at least $10 \mathrm{TeV}$. Firm identification of the nature of such sources is quite important since they can provide information to understand a long-standing question in science, the origin of the highest energy CRs up to PeV energies.

In this work, we present dedicated VHE and multi-wavelength (MWL) data analysis results and interpretation of three intriguing H.E.S.S. sources, HESS J1641-463 [3], HESS J1741-302 [4] and HESS J1826-130 [5]. These three H.E.S.S. sources show common characteristics, especially from the spectral point of view, as listed below.

- Inspection of their VHE $\gamma$-ray spectra suggests that the astrophysical objects producing such emission must be capable of accelerating the parental particle population up to energies of at least several hundreds of $\mathrm{TeV}$.

- All of them are tagged as unidentified sources and located near other bright VHE sources, thus their (apparent) emission is contaminated and suffering from source confusion.

- Their best fit positions and extensions are spatially coincident with dense gas regions.

- None of the sources shows variable VHE emission.

\section{H.E.S.S. OBSERVATIONS and RESULTS}

\subsection{The H.E.S.S. Telescopes}

The High Energy Stereoscopic System (H.E.S.S.) is an array of five imaging atmospheric Cherenkov telescopes located in the Khomas Highland of Namibia, $1800 \mathrm{~m}$ above sea level. H.E.S.S. in phase I comprised of four $13 \mathrm{~m}$ diameter telescopes which have been fully operational since 2004. A fifth telescope was added in the center of the array and has been operational since September 2012. The H.E.S.S. phase I array configuration is sensitive to $\gamma$-ray energies between $100 \mathrm{GeV}$ and several tens of TeV. With the addition of the fifth telescope, the energy threshold was lowered down to some tens of GeV. The VHE H.E.S.S. data presented in this paper were taken with the H.E.S.S. phase I array configuration, which can measure extensive air showers with an angular resolution better than $0.1^{\circ}$ and an energy resolution of $15 \%$ at an energy of $1 \mathrm{TeV}$ [6]. 


\subsection{H.E.S.S. Analysis Details}

The data presented here have been analyzed with the H.E.S.S. analysis package for shower reconstruction. For the case of HESS J1741-302 and HESS J1826-130, the multivariate analysis (TMVA) technique [7] has been applied for providing the best available rejection power between hadrons and $\gamma$ rays, while this technique has not been applied for the case of HESS J1641-463. The CR background levels were estimated using the ring background model [8] for source detection and morphology studies. The detection significances were determined by using Equation (17) in [9]. The differential VHE $\gamma$-ray spectra of these sources were derived using the forward folding technique [10] and the reflected background model [8] was used for the background estimation. A log-likelihood ratio test (LLRT) was used for comparing both the morphology and spectral models and deciding the preferred models that describe the data best.

\subsection{Morphology Results}

Thanks to the excellent sensitivity of H.E.S.S., combined with the high density of sources in the Galaxy, the number of detected sources increases significantly. But this leads to confusion of sources, especially for the ones located in the vicinity of bright sources. All three sources presented here suffer from source confusion in different levels, which means that they are contaminated by the emission coming from nearby bright sources.

Note that for such sources suffering from source confusion, an investigation in different energy bands can provide an additional powerful tool for new discoveries [3]. Such an approach has been applied for the cases of HESS J1641 -463 and HESS J1826-130, since these two sources were hidden under the tails of the bright VHE emission from HESS J1640-465 [11] and HESS J1825-137 [12], respectively. The situation is similar for the case of HESS J1741-302, but since the nearby bright source, HESS J1745-303 [13], is located farther with respect to the HESS J1641-463 and HESS J1826-130 cases, HESS J1741-302 is less affected by the source confusion. Figures 1a and Fig. 1b show how the visibility of both HESS J1641-463 and HESS J1826-130 becomes more prominent as the energy threshold is increased.

A hard cut configuration was used to optimize the angular resolution, including a minimum of 200 photoelectrons (p.e.) cut for HESS J1641-463, and 160 p.e for the other two sources. The excess maps were fitted by 2D Gaussian models, convolved with the H.E.S.S. point spread function (PSF). The morphological analysis results of these three sources are given in Table 1.

\subsection{Spectral Results}

Source confusion does not only affect source morphologies, but may also potentially distort the observed spectra of VHE $\gamma$-ray sources. This effect is negligible $(<10 \%)$ for the case of HESS J1741-302 when hard cut configuration is used. In the case of HESS J1641-463, the spectral contamination was estimated $\sim 15 \%$ above $0.64 \mathrm{TeV}$, while it is reduced to $\sim 4 \%$ above 4.0 TeV. Finally, HESS J1826-130 is the most contaminated VHE source in all three sources. The preliminary spectral contamination estimation gives $\sim 40 \%$ below $1.5 \mathrm{TeV}$ and $\sim 20 \%$ above 1.5 $\mathrm{TeV}$. Spectral properties of the sources ${ }^{1}$ presented here are given in Table 2 and are affected by

\footnotetext{
${ }^{1}$ HESS J1826-130 spectral parameters are updated with respect to previously published results [5] taking into account the updated value of the mirror reflection modelling in H.E.S.S. telescopes.
} 


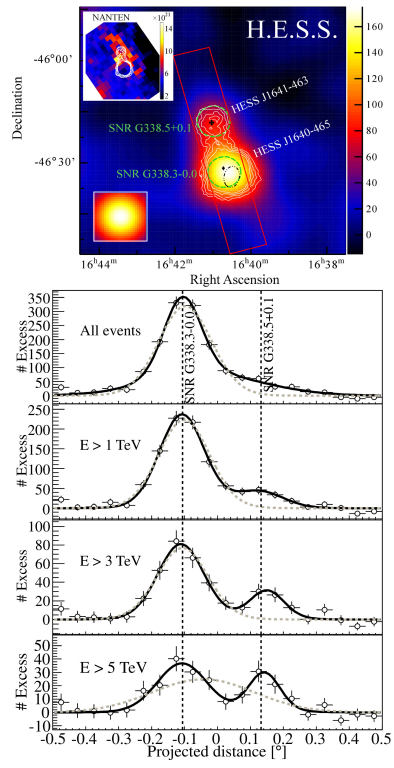

(a)
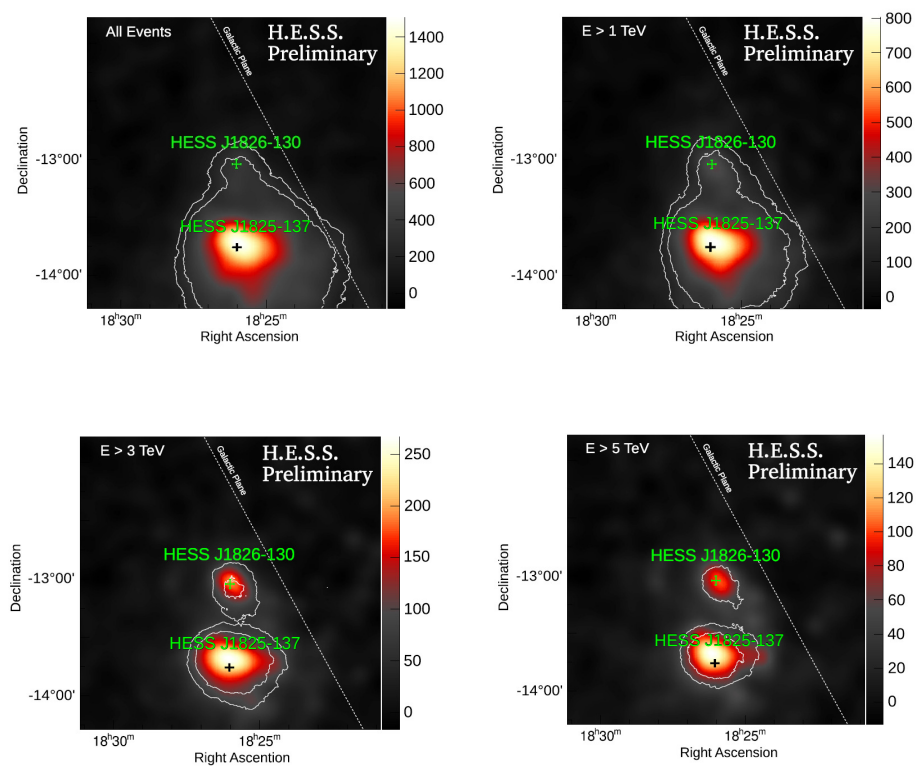

(b)

Figure 1: (a) Energy dependent excess slices of HESS J1641-463, the upper panel showing the excess map and the lower panel the distribution of VHE excess events, extracted from the red rectangle of the upper panel, and corresponding Gaussian fits in different energy bands. The figures are from [3]. (b) Energy dependent maps of HESS J1826-130 excess maps for all events and E > 1,3 and $5 \mathrm{TeV}$. The white contours indicate the emission regions at $15 \sigma$ and $20 \sigma$ level. The figures are from [5].

\begin{tabular}{|c|c|c|c|c|c|c|}
\hline Source Name & Observation Period & $\begin{array}{c}\text { Live-time } \\
\mathrm{h}\end{array}$ & $\begin{array}{c}\text { Significance } \\
\sigma\end{array}$ & $\begin{array}{c}\text { Best Fit Position } \\
\text { (J2000) }\end{array}$ & $\begin{array}{c}\text { Extension } \\
\left({ }^{\circ}\right)\end{array}$ & Source Morphology \\
\hline $\begin{array}{l}\text { HESS J1641-463 } \\
(\mathrm{E}>4.0 \mathrm{TeV})\end{array}$ & $2004-2011$ & 72 & 8.5 & $\begin{array}{c}\text { R.A.: } 16^{\mathrm{h}} 41^{\mathrm{m}} 2.1^{\mathrm{s}} \\
\text { Dec.: }-46^{\circ} 18^{\prime} 13.0^{\prime \prime}\end{array}$ & $\begin{array}{l}0.050 \\
\text { (UL) }\end{array}$ & Point-like \\
\hline $\begin{array}{l}\text { HESS J1741-302 } \\
(\mathrm{E}>0.4 \mathrm{TeV})\end{array}$ & $2004-2013$ & 145 & 7.8 & $\begin{array}{l}\text { R.A.: } 17^{\mathrm{h}} 41^{\mathrm{m}} 15.8^{\mathrm{s}} \\
\text { Dec.: }-30^{\circ} 22^{\prime} 30.7^{\prime \prime}\end{array}$ & $\begin{array}{l}0.077 \\
\text { (UL) }\end{array}$ & Point-like \\
\hline $\begin{array}{l}\text { HESS J1826-130 } \\
(\mathrm{E}>0.5 \mathrm{TeV})\end{array}$ & $2004-2015$ & 204 & 21.0 & $\begin{array}{l}\text { R.A.: } 18^{\mathrm{h}} 26^{\mathrm{m}} 0.2^{\mathrm{h}} \\
\text { Dec.: }-13^{\circ} 02^{\prime} 1.8^{\prime \prime}\end{array}$ & $0.17 \pm 0.02$ & Extended \\
\hline
\end{tabular}

Table 1: Morphology analyses results of the sources. The column labelled as observation period shows the year time of the observations, while the live-time column gives the corresponding acceptance corrected live-time of the data. The significance columns gives the detection significance of the source. Note that the detection significance and morphology model parameters for HESS J1641-463 are given for the energies above 4.0 TeV. The columns labelled as best fit position and extension give the source morphology model parameters obtained from morphology analyses, while the source morphology column indicates whether the source size is compatible with (pointlike) or significantly larger (extended) than the H.E.S.S. PSF for the corresponding analysis. For the point-like sources, the $99 \%$ upper limits (UL) on the extension are given. 
contamination. The intrinsic (uncontaminated) spectrum of HESS J1826-130 is expected to be even harder (or having a cut-off at higher energies), given the relatively softer spectrum of contaminating emission from HESS J1825-137. Our preliminary results provide an intrinsic spectrum of HESS J1826-130 with a spectral index of $\Gamma=1.57 \pm 0.15_{\text {stat }}$ and a cut-off at $15.2_{-4.1}^{+8.9} \mathrm{TeV}$.

\begin{tabular}{cccccc}
\hline \hline Source Name & Spectral Model & $\begin{array}{c}\text { Normalization (at } 1 \mathrm{TeV}) \\
10^{-13} \times \mathrm{cm}^{-2} \mathrm{~s}^{-1} \mathrm{TeV}^{-1}\end{array}$ & Index & $\begin{array}{c}\text { Cut-off Energy } \\
(\mathrm{TeV})\end{array}$ & $\begin{array}{c}\text { Flux }(>1 \mathrm{TeV}) \\
(\mathrm{Crab} \text { Unit } \%)\end{array}$ \\
\hline HESS J1641-463 & PL & $3.91 \pm 0.69_{\text {stat }} \pm 0.8_{\text {sys }}$ & $2.07 \pm 0.11_{\text {stat }} \pm 0.20_{\text {sys }}$ & - & 1.8 \\
\hline HESS J1741-302 & PL & $2.1 \pm 0.4_{\text {stat }} \pm 0.4_{\text {sys }}$ & $2.3 \pm 0.2_{\text {stat }} \pm 0.2_{\text {sys }}$ & - & 1.0 \\
\hline HESS J1826-130 & ECPL & $8.28 \pm 0.68_{\text {stat }} \pm 1.6_{\text {sys }}$ & $1.66 \pm 0.11_{\text {stat }} \pm 0.20_{\text {sys }}$ & $13.5_{-2.7}^{+4.7}$ & 4.0
\end{tabular}

Table 2: Spectral parameters of the sources presented in this work. The column labelled as spectral model indicates whether the preferred spectral model of the source is a simple power-law function (PL) or a power-law with an exponential cut-off (ECPL) function. The normalization, index and cut-off energy columns give the corresponding model parameters, while the flux column gives the corresponding integrated flux above $1 \mathrm{TeV}$ in the units of Crab Nebula flux above same energy.

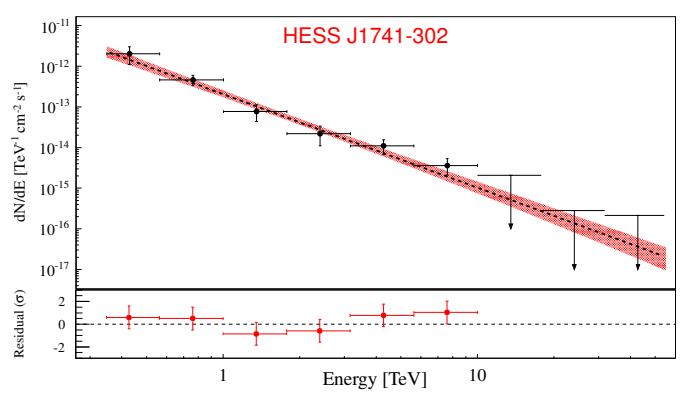

(a)

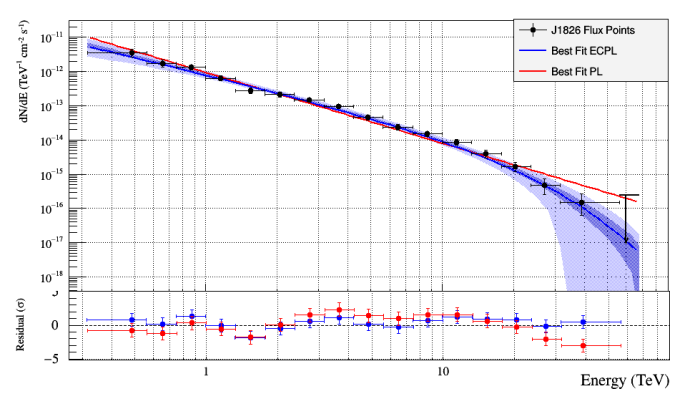

(b)

Figure 2: VHE $\gamma$-ray spectra of HESS J1741-302 [14] (a) and HESS J1826-130 [5] (b). The red and blue shaded areas represent the $68 \%$ confidence interval for the fitted spectral model of PL (a) and ECPL (b), while the light blue shaded region represents the $99 \%$ confidence interval of the ECPL model.

\subsection{Interstellar Medium}

A characteristic shared among these three sources is that there are dense gas regions coincident with their best fit positions. These dense gas regions can provide rich target material for accelerated particles to produce VHE $\gamma$-ray emission via proton-proton interactions followed by a subsequent $\pi^{0}$ decay [15], especially in case a CR accelerator (such as a SNR) is located close to, or coincident with, the sources of interest. For investigating a possible hadronic origin of the emission detected from these sources, the analysis of data from available surveys of atomic and molecular hydrogen around the location of the sources have been carried out. The distribution of molecular gas around 
the sources presented in this work is obtained by integrating the ${ }^{12} \mathrm{CO} 1 \rightarrow 0$ rotational line emission measured with the NANTEN Sub-millimeter Observatory [16].

The distribution of molecular gas around HESS J1641-463 is obtained by integrating over a range in velocity between $-40 \mathrm{~km} \mathrm{~s}^{-1}$ to $-30 \mathrm{~km} \mathrm{~s}^{-1}$. The total column density from the extraction region of the source is $1.7 \times 10^{22} \mathrm{~cm}^{-2}$, while the density and the total mass are about $100 \mathrm{~cm}^{-3}$ and $2.4 \times 10^{5}$ solar masses at $11 \mathrm{kpc}$, respectively [3]. In the case of HESS J1741-302, eight molecular clouds (MCs) found coincident with the best fit position of the source along the line of sight. Integration velocity intervals change between $+7 \mathrm{~km} \mathrm{~s}^{-1}$ and $-221 \mathrm{~km} \mathrm{~s}^{-1}$ depending on the corresponding molecular cloud (MC). Analysis of these MCs give the densities and total masses changing in the range of $[62,380] \mathrm{cm}^{-3}$ and $[1.9,9.8] \times 10^{5}$ solar masses at the corresponding MC distances, respectively (see [14] for the details of the interstellar medium study). The analysis of interstellar medium around HESS J1826-130 points out two giant molecular clouds (GMC) with few $10^{5}$ solar masses at $3.7 \mathrm{kpc}$ and $4.7 \mathrm{kpc}$ [17]. The ambient gas densities of these GMCs are estimated of the order of $600 \mathrm{~cm}^{-3}$.

\section{DISCUSSIONS}

Investigation of VHE $\gamma$-ray sources showing hard spectrum without a clear cut-off is important to understand the origin of the highest energy CRs close to the knee in our Milky Way Galaxy, especially in the presence of dense gas regions coincident with the sources. The sources presented in this work show common characteristics in this sense, thus indicating possible hadronic VHE emission origin. Assuming that the VHE emission originates from hadronic processes happening in the dense gas regions [18] and taking into account the properties of these regions coincident with these sources, one can conclude that the spectra of parental particle population can extend up to at least several hundreds of TeV. The total energy required in protons, $W_{p p}$, to produce the inferred $\gamma$ ray luminosity, $L_{\gamma}=4 \pi F_{\gamma}(>0.4 \mathrm{TeV}) D^{2}$, can be estimated as $W_{p p}=L_{\gamma} \times t_{p p}$, where $t_{p p}=5.76 \times$ $10^{15} \times\left(n_{\text {gas }} / \mathrm{cm}^{-3}\right)^{-1} \mathrm{~s}$ is the cooling time for proton-proton collisions. Calculations of the energy budget for these sources gives that $W_{p p}=\sim 10^{48}$ erg and $W_{p p}=\sim 10^{47}$ erg for HESS J1641-463 [3] and HESS J1826-130 [5], for the distances of $11 \mathrm{kpc}$ and $4 \mathrm{kpc}$, respectively. In the case of HESS J1741-302, $W_{p p}$ is found between $7.0 \times 10^{46} \mathrm{erg}$ and $1.5 \times 10^{48} \mathrm{erg}$ depending on gas densities calculated for each MC along the line of sight [14].

In any case, our studies do not allow us to distinguish whether the VHE $\gamma$-ray emission from these sources has leptonic or hadronic origin. Taking into account the point-like morphologies of HESS J1641-463 and HESS J1741-302, binary scenarios can also be envisaged. The fact that no variability could be observed from these sources can not be taken as evidence for disfavoring binary origin because of the low statistics, while the lack of an optical counterpart can be related to high optical extinction or the location of sources close to the Galactic plane. One can not exclude a cut-off in the $\gamma$-ray spectra of these two sources because of the limited statistics above $10 \mathrm{TeV}$. In addition, a leptonic scenario, where electrons accelerated by the pulsar PSR J1826-1256 are up-scattering CMB or IR photons, can also explain the VHE emission from HESS J1826-130. Such as hard spectrum at H.E.S.S. energies can be produced by an uncooled electron population with spectral index close to 2.0 and a cut-off at around $70 \mathrm{TeV}$. The energy output in accelerated electrons is $2 \times 10^{47} \mathrm{ergs}$ for a distance of $7 \mathrm{kpc}$. This source has a spectrum very similar to other 
PWNe, in particular, Vela X [19]. HESS J1826-130 could be an indication of a distinctive PWN population, with very hard spectra and relatively high cut-off energies

\section{CONCLUSIONS}

The analysis of the three sources presented here shows the common characteristic of very hard spectra and plausible scenarios where parental population of hadrons extend up to several hundreds of $\mathrm{TeV}$, suggesting they may be representing a population of $\mathrm{CR}$ accelerators active in the Galaxy. However, an interpretation based on leptonic scenarios can not be discarded.

The future Cherenkov Telescope Array (CTA), with its much better angular and energy resolution and sensitivity, with respect to the current imaging air Cherenkov telescope systems, will facilitate a deeper study and better constrain the spectral parameters of these sources . In addition, CTA will be able to detect many more VHE $\gamma$-ray sources throughout the Galaxy, some of them perhaps sharing characteristics with the sources presented here. In conclusion, the enhanced capabilities of CTA will allow us to establish if such a population of CR accelerators active in the Galaxy.

\section{ACKNOWLEDGEMENTS}

The support of the Namibian authorities and of the University of Namibia in facilitating the construction and operation of H.E.S.S. is gratefully acknowledged, as is the support by the German Ministry for Education and Research (BMBF), the Max Planck Society, the German Research Foundation (DFG), the French Ministry for Research, the CNRS-IN2P3 and the Astroparticle Interdisciplinary Programme of the CNRS, the U.K. Science and Technology Facilities Council (STFC), the IPNP of the Charles University, the Czech Science Foundation, the Polish Ministry of Science and Higher Education, the South African Department of Science and Technology and National Research Foundation, the University of Namibia, the Innsbruck University, the Austrian Science Fund (FWF), and the Austrian Federal Ministry for Science, Research and Economy, and by the University of Adelaide and the Australian Research Council. We appreciate the excellent work of the technical support staff in Berlin, Durham, Hamburg, Heidelberg, Palaiseau, Paris, Saclay, and in Namibia in the construction and operation of the equipment. This work benefited from services provided by the H.E.S.S. Virtual Organisation, supported by the national resource providers of the EGI Federation. This research has made use of software provided by the Chandra X-ray Center (CXC) in the application packages CIAO, ChIPS, and Sherpa. This research has made use of the SIMBAD database, operated at CDS, Strasbourg, France. This research has made use of the ATNF pulsar catalog database (http://www.atnf.csiro.au/research/pulsar/psrcat/). The NANTEN project is based on the mutual agreement between Nagoya University and the Carnegie Institution of Washington. Sabrina Casanova and Ekrem Oğuzhan Angüner acknowledge the support from the Polish National Science Center under the Opus Grant UMO-2014/13/B/ST9/00945.

\section{References}

[1] H.E.S.S. Collaboration, H. Abdalla, A. Abramowski, F. Aharonian, F. Ait Benkhali, and A. Akhperjanian, Nature, 531, 476 (2016) 
[2] H.E.S.S. Collaboration, H. Abdalla, A. Abramowski, F. Aharonian, F. Ait Benkhali, and A. Akhperjanian, The H.E.S.S. Galactic Plane Survey, A\&A forthcoming (2017).

[3] A. Abramowski et al., The Astrophysical Journal Letters, 794:L1 (2014)

[4] O. Tibolla, N. Komin, K. Kosack, M. Naumann-Godo, AIP Conf. Proc., 1085, 1 (2008)

[5] E. O. Angüner et al., AIP Conf. Proc., 1792, 1 (2017)

[6] F. A. Aharonian et al., A\&A, 457, 899 (2006)

[7] S. Ohm, C. van Eldik and K. Egberts, AP, 31, 383 (2009)

[8] D. Berge, S. Funk and J. Hinton, A\&A, 466, 1219 (2007)

[9] T. -P. Li and Y. -Q. Ma, ApJ, 272, 317 (1983)

[10] F. Piron, A. Djannati-Atai and M. Punch, A\&A, 374, 895 (2001)

[11] A. Abramowski, F. Aharonian, F. A. Benkhali et al., MNRAS, 439, 2828 (2014)

[12] F. Aharonian et al., A\&A, 442, 25-29 (2005)

[13] F. Aharonian et al., ApJ, 636, 2 (2005)

[14] H.E.S.S. Collaboration, H. Abdalla, A. Abramowski, F. Aharonian, F. Ait Benkhali, and A. Akhperjanian, HESS J1741-302: a hidden accelerator in the Galactic plane, A\&A forthcoming (2017).

[15] F. Aharonian, ApSS, 180, 2 (1991)

[16] A. Mizuno, Y. Fukui, Proc. of ASP Conference, 317, 59 (2004)

[17] F. Voisin, G. Rowell, M. G. Burton et al., MNRAS, 458, 2813-2835 (2016)

[18] S. R. Kelner, F. Aharonian, \& V. V. Bugayov, Phys. Rev. D, 74, 034018 (2006)

[19] F. Aharonian et al., A\&A, 448, 43 (2006) 